\title{
Analysis of resonant fast ion distributions during combined ICRF and NBI heating with transients using neutron emission spectroscopy
}

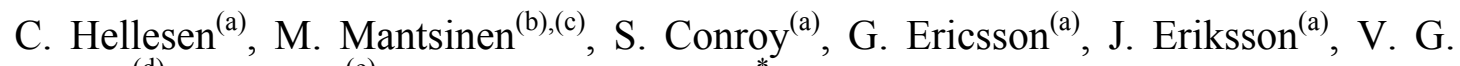
Kiptily $^{(\mathrm{d})}$, F. Nabais ${ }^{(\mathrm{e})}$, and JET Contributors ${ }^{*}$

EUROfusion Consortium, JET, Culham Science Centre, Abingdon, OX14 3DB, UK

(a) Department of Physics and Astronomy, Uppsala University, Uppsala, Sweden

(b) Barcelona Supercomputing Center (BSC)

(c) ICREA, Pg. Lluís Companys 23, 08010 Barcelona, Spain.

(d) CCFE, Culham Science Centre, Abingdon, Oxfordshire OX14 3DB, UK

(e) IST, Centro de Fusao Nuclear, Lisbon, Portugal

E-mail:carl.hellesen@physics.uu.se

\section{Abstract}

This paper studies the deuterium distributions from $2^{\text {nd }}$ harmonic ICRF heating at JET using neutron emission spectroscopy data from the time of flight spectrometer TOFOR. The fast deuterium distributions are obtained over the energy range $100 \mathrm{keV}$ to $2 \mathrm{MeV}$. Specifically, we study how the how the fast deuterium distributions vary as ICRF heating is used alone as well as in combination with NBI heating.

When comparing the different heating scenarios we observed both a difference in the slope of the distributions as well as in their absolute level. In the case of ICRF heating alone, the slope is Maxwellian-like over the entire energy range studied, while for the combined ICRF + NBI heating we observe a non-Maxwellian slope, which flattens out above $0.5 \mathrm{MeV}$. Comparisons are made with corresponding distributions calculated with the code PION. We find a good agreement between the measured distributions and those calculated with PION, both in terms of their shapes as well as their amplitudes.

However, we also identified a period with an inverted fast ion distribution, which showed large disagreements between the modeled and measured results. Resonant interactions with tornado modes, i.e. core localized Toroidal Alfven Eigenmodes, are put forward as a likely explanation for the inverted distribution.

\section{Introduction}

The heating of a magnetically confined fusion plasma to the temperatures required for ignition is mainly foreseen to be accomplished using auxiliary heating systems, i.e. neutral beam injection (NBI) heating and ion cyclotron radio frequency (ICRF) heating. Both means of plasma heating involve the creation of a sub population of energetic ions that transfer energy to the bulk plasma through collisions. ICRF heating works by tuning a microwave to the ion cyclotron frequency $\left(\omega_{\mathrm{c}}\right)$, or a harmonic $\left(\omega_{\mathrm{ICRF}}=n \omega_{\mathrm{c}}\right)$, of one of the plasma ion species, where $n$ is the harmonic number.

\footnotetext{
"See the author list of "Overview of the JET results in support to ITER" by X. Litaudon et al. to be published in Nuclear Fusion Special issue: overview and summary reports from the 26th Fusion Energy Conference (Kyoto, Japan, 17-22 October 2016)
} 
Fundamental $(n=1)$ ICRF heating cannot be used to heat a majority fuel ion species due to the unfavorable polarization of the wave at the resonance layer. Therefore, it is not possible to directly heat the fuel ions, deuterium (D) and tritium (T), in a burning D-T plasma with fundamental ICRF heating. Instead, a minority ion species that can absorb the ICRF power has to be included. This is typically hydrogen or He-3, and the concentrations are usually around a few percent of the majority concentration.

While harmonic ICRF heating $(n>1)$ can be used to directly heat a majority fuel ion species in the plasma, it is a finite Larmor radius process, which is rather inefficient for heating a cold ion population. For efficient harmonic ICRF heating, the resonant ion species must have a population of energetic ions that the ICRF wave can couple to. This could be either the wings of a high temperature thermal population, or the slowing down distribution from NBI heating. Illustrative examples from the Joint European Torus (JET) can be found in [L.-G. Eriksson 1998], [Mantsinen 2002a], [Kiptily 2005] and [Hellesen 2013]. In the former a fast deuterium population was created with $3^{\text {rd }}$ harmonic ICRF heating, and as a tail of fast deuterium was gradually built up the ICRF absorption was improved. In the three latter, $3^{\text {rd }}$ harmonic ICRF heating was combined with NBI heating, which resulted in a strong synergetic effect. High harmonic heating has also been tested at other tokamaks, e.g., DIII-D [Mantsinen, 2002b] as well as ASDEX [Mantsinen 2016].

Fundamental heating of a minority ion species and $2^{\text {nd }}$ harmonic heating of a fuel ion species often coincide. Two examples are fundamental hydrogen and $2^{\text {nd }}$ harmonic deuterium heating as well as fundamental He- 3 and $2^{\text {nd }}$ harmonic tritium heating. The former is typically used at JET, and the latter is the main ICRF heating scenario for burning plasmas at ITER [ITER 1999]. It was also tested during the JET DTE1 experiment [Start 1999]. In both scenarios, damping by the two resonant species together with direct electron damping are competing power absorption mechanisms. The resulting power partition is strongly dependent on the minority concentration as well as the temperature of the majority population, where a higher temperature results in increased power absorption of the majority species. It has been proposed [L.-G. Eriksson 1999] that fundamental minority He-3 heating is used in a fusion reactor during the startup phase of a plasma, before the fuel ion temperature is high enough to enable efficient $2^{\text {nd }}$ harmonic tritium heating. Once the required fuel ion temperature is reached, no more He-3 is added to the plasma, and the heating gradually turns to $2^{\text {nd }}$ harmonic tritium heating. It is desirable to avoid the presence of a minority $\mathrm{He}-3$ population during the burn phase as each He-3 ion occupies the same charge as two fuel ions. For example, a He-3 concentration of 5\% would reduce the fuel ion density by $10 \%$, which would in turn lower the fusion power by $20 \%$. Access to reliable modeling of the ICRF power competition between the majority and minority fuel ion species at different temperatures is therefore important when optimizing the performance of future burning plasma scenarios, e.g. at ITER.

The presence of supra-thermal fuel ions from NBI heating can also increase the effective temperature of the fuel ions and therefore affect the power partition. In a relatively cold plasma, where the harmonic absorption is expected to be poor, a strong synergetic effect between harmonic ICRF heating and NBI heating is expected. The slowing down population from the neutral beam provides a seed of supra thermal ions that the ICRF wave can couple to. Such synergetic effects between NBI and harmonic ICRF heating NBI have been shown e.g. in [Mantsinen 2002a] and [Hellesen 2013]. 
This effect is the focus of this paper. We study in detail how the NBI+ICRF synergy affects the power partition between the majority and minority fuel ion species using time resolved measurement of the fast deuterium distribution function. The deuterium distribution function was obtained by analyzing the neutron spectrum from the TOFOR time-of-flight spectrometer [Gatu 2008]. The measured distributions are subsequently compared with calculated distribution functions using the code PION [L.-G. Eriksson 1993], which takes into account the NBI+ICRF synergy [Mantsinen 1999]. We selected an ICRF heated plasma at JET with two 2-second long blips of NBI heating. This allowed us to dynamically study how the fast deuterium distribution changed as the seed population of fast deuterons from NBI heating was turned off and subsequently turned on again. Meanwhile, the ICRF heating power remained constant. Furthermore, we also examine the effect that resonances between fast ions and Magneto Hydro Dynamic activities can have. Such phenomena are not modeled by the PION code and leads to discrepancies in the comparison.

\section{Materials and methods}

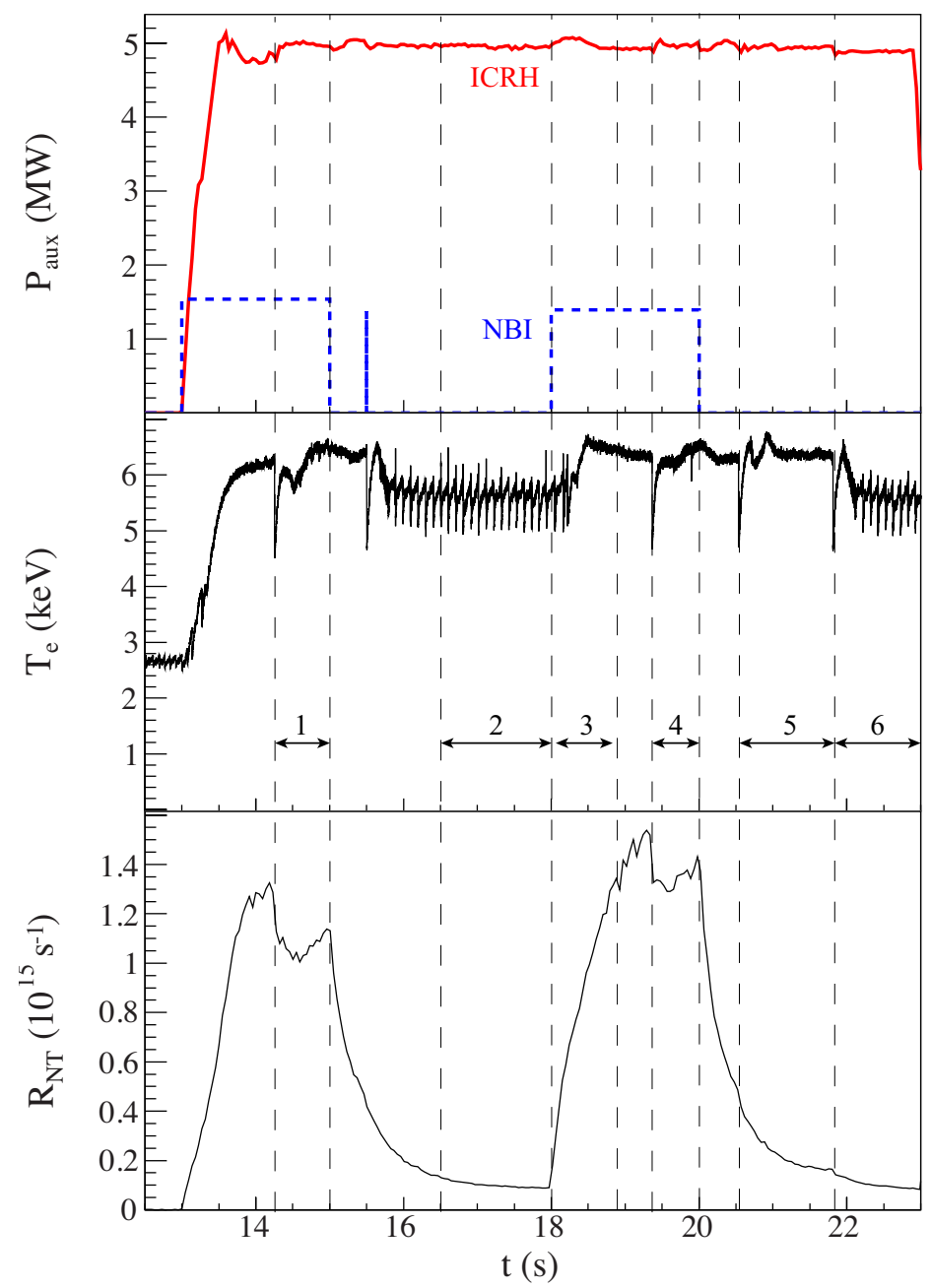

Figure 1. Time evolution of auxiliary heating power (a), core electron temperature (b) as well as total neutron rate (c) for JET discharge \#69247. 


\subsection{Plasma scenario}

In this paper we study JET discharge \#69247 as it was identified to be a good candidate for isolating the synergetic effects between NBI and ICRF heating. This discharge was run in L-mode with a monotonic q-profile and heated by $5 \mathrm{MW}$ of ICRF power (H-minority). In addition, it also had two phases with 1.5 MW of NBI heating, which lasted for $2 \mathrm{~s}$ each. The time evolution of the auxiliary heating is shown in figure 1 (a). The plasma electron density was around $2 \cdot 10^{19} \mathrm{~m}^{3}$.

The toroidal magnetic field at the magnetic axis was $B_{0}=2.75 \mathrm{~T}$, and the ICRF heating was tuned at $f=42 \mathrm{MHz}$, which places the fundamental hydrogen and $2^{\text {nd }}$ harmonic deuterium resonances close to the magnetic axis at $R=3.0 \mathrm{~m}$. The NBI injection energy during the first blip was $76 \mathrm{keV}$ and the injection energy during the second blip was $127 \mathrm{keV}$. The modulation of the NBI heating allows for studying how the fast deuterium distribution changes going from ICRF heating only to combined NBI + ICRF heating.

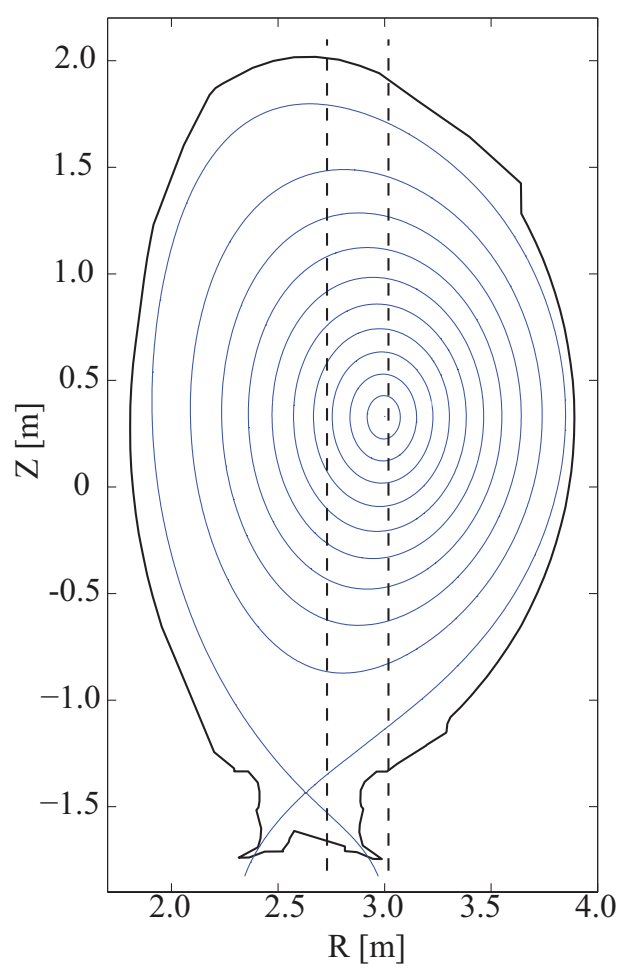

Figure 2. Poloidal cross section of JET. The solid black line mark the first wall of the tokamak, the blue lines indicate contours of constant magnetic flux for a given plasma equilibrium and the dashed black lines mark the edges of the field of view of TOFOR.

\subsection{Neutron analysis}

Neutron spectroscopy data were taken using the TOFOR neutron time-of-flight spectrometer [Gatu 2008]. TOFOR is installed in the roof laboratory at JET and views the plasma core from above with a field of view that is perpendicular to the magnetic field. See Figure 2 for an illustration.

The fast deuterium distribution functions were found using the method described in [Hellesen, 2013]. In short, pre calculated neutron energy spectra from mono-energetic deuterium distributions ( $\delta$-spectra) are multiplied with the spectrometer response 
function and their sum was fitted to the measured data. The $\delta$-spectra can be seen as the "building blocks", or a basis set, that maps a distribution function in deuterium energy to TOFOR data. The resulting weight of each $\delta$-spectrum in the fit can then be used to retrieve the level of the deuterium distribution function at the corresponding energy.

In addition to the deuteron energy, the shapes of the $\delta$-spectra also depend on the pitch, i.e., the direction of the velocity vector relative to the magnetic field $\left(\xi=\mathrm{v}_{\|} / \mathrm{v}\right)$. However, since neutron spectroscopy data is here only taken along one sightline it is not possible, in practice, to obtain any information on the pitch distribution of the fast ions from the measured data. Instead, an assumption on the pitch distribution must be made when calculating the $\delta$-spectra. In [Hellesen 2013], a narrow distribution centered at $\xi=0$ was used. This was based on the fact that ICRF heating accelerates ions in the direction perpendicular to the magnetic field, and as their energy is increased they approach $\xi=0$. In this paper, the analysis with $\delta$-spectra is refined by defining an energy dependent pitch distribution in the following way. We start with the normalized magnetic moment of a particle $(\Lambda)$, which is related to the magnetic moment $\left(\mu=m v^{2}{ }_{\perp} / 2 B\right)$ and the kinetic energy $(E)$ through

$$
\Lambda=\frac{B_{0}}{E} \mu=\frac{R}{R_{0}}\left(1-\xi^{2}\right)
$$

where $R$ is the location of the particle along the major radius, and $R_{0}$ is the major radius of the tokamak. The rightmost hand side of this equation holds in the limit of a large aspect ratio tokamak (such as JET), where $B \approx B_{0} R_{0} / R$. When ions are heated by ICRF they follow, to first order, trajectories in phase space along ion cyclotron characteristics given by [Hellsten 2004 and Johnson 2016]

$$
\Delta \Lambda=\frac{\Lambda_{\mathrm{res}}-\Lambda}{E+\Delta E} \Delta E
$$

where $\Lambda_{\text {res }}=n_{\mathrm{ic}} \omega_{\mathrm{c} 0} / \omega_{c}$ is the cold ion cyclotron resonance position. Here $n_{\mathrm{ic}}$ is the ion cyclotron harmonic, $\omega_{\mathrm{c} 0}$ is the ion cyclotron frequency at the magnetic axis and $\omega_{\mathrm{c}}$ is the cyclotron frequency at the resonance. A TRANSP simulation of JET \#69242 [Hellesen 2010a], which had the same NBI heating as JET \#69247, but no ICRF heating, shows that the NBI ions are deposited in the plasma core with $\xi=0.6$. This gives $\Lambda=0.64$, and assuming a similar deposition profile for $\# 69247$, an energy dependent $\Lambda$ distribution is found for ions accelerated above the NBI energy using equation (2). Since the beam ions are deposited in the plasma core we have $R=R_{0}$ and the pitch as a function of energy can then be obtained from $\xi(E)=\sqrt{ } 1-\Lambda(E)$. This forms the basis for calculating the $\delta$-spectra in this paper when combined NBI and ICRF heating was used. For the time periods with pure ICRF heating, the pitch was instead assumed to be centered at $\xi=0$ since the ions are accelerated from the isotropic thermal distribution.

Care needs to be taken when choosing the appropriate number of $\delta$-spectra as well as their spacing on the ion energy scale. If the $\delta$-spectra are positioned too closely their resulting representation in the data will to a large degree overlap, and the fitting 
problem becomes ill posed. This means that there is no longer a unique solution to the problem. This can be overcome by regularization, which is the method pursued in e.g. [Salewski 2017]. In this paper the approach is instead the opposite, the number of $\delta$ spectra are limited such that they span a near orthogonal basis set, .i.e., the $\delta$-spectra are to a large degree non-overlapping. The problem therefore remains well posed. 6 suitable spectra were identified at $E_{\mathrm{d}}=100,300,600,1000,1500$ and $2000 \mathrm{keV}$. These energies were used for pure ICRF heating as well as for combined NBI + ICRF heating with $76 \mathrm{keV} \mathrm{NBI}$ injection energy. In the case where the NBI injection energy was set to $127 \mathrm{keV}$, the lowest energy for the $\delta$-spectra was increased to $150 \mathrm{keV}$. In this way, the analyzed region is always above the NBI injection energy, and we are guaranteed that the results we obtain are due to acceleration of ions above the NBI injection energy.

\section{Results and discussion}

In figure 2 we show the time evolution of the auxiliary plasma heating (a), central electron temperature (b) and total neutron rate (c). Already from the total neutron rate we can see a clear synergetic effect between the NBI and ICRF heating systems. As 1.6 MW of NBI heating is added to $5 \mathrm{MW}$ of ICRF heating the neutron rate increases more than tenfold from about $10^{14} \mathrm{~s}^{-1}$ to $1.4 \cdot 10^{15} \mathrm{~s}^{-1}$. It should be pointed out that this is not only a result of NBI heating being more efficient at producing neutrons than ICRF heating. In discharge \#69242, an identical NBI blip (18-20 s) was used, but with no ICRF heating. This resulted in a neutron emission of $3.2 \cdot 10^{15} \mathrm{~s}^{-1}$, or about $1 / 4$ of the combined ICRF+NBI heating. The combination of NBI+ICRF heating is here significantly larger than the sum of their individual contributions.

Six time windows are also marked in figure 2. These are used as integration periods for the analysis of TOFOR data. The boundaries of the time windows are chosen either to represent periods of relatively stable neutron emission (time windows 1 and 4), the transient phase when the NBI heating is switched on (time window 3), or the decay of the fast ion population as the NBI heating is switched off (time windows 2, 5 and 6). Moreover, we do not integrate over any monster sawteeth (seen in the electron temperature) since sawtooth physics is not a part of the PION modeling presented in this paper.

In figure 3, examples of TOFOR data from time windows 4 and 5 are shown in panels (a) and (b), respectively. These intervals represent combined NBI+ICRF heating (time window 4) as well as ICRF heating alone (time window 5). We see here that in both cases a large part of the neutron emission is manifested at $t_{\mathrm{TOF}}<60 \mathrm{~ns}$, which is a signature of deuterons being accelerated beyond the NBI injection energy, c.f. [Hellesen et al. 2010]. We also show the fitted $\delta$-spectra used to obtain the fast deuterium distribution function (thin full drawn gray lines). 

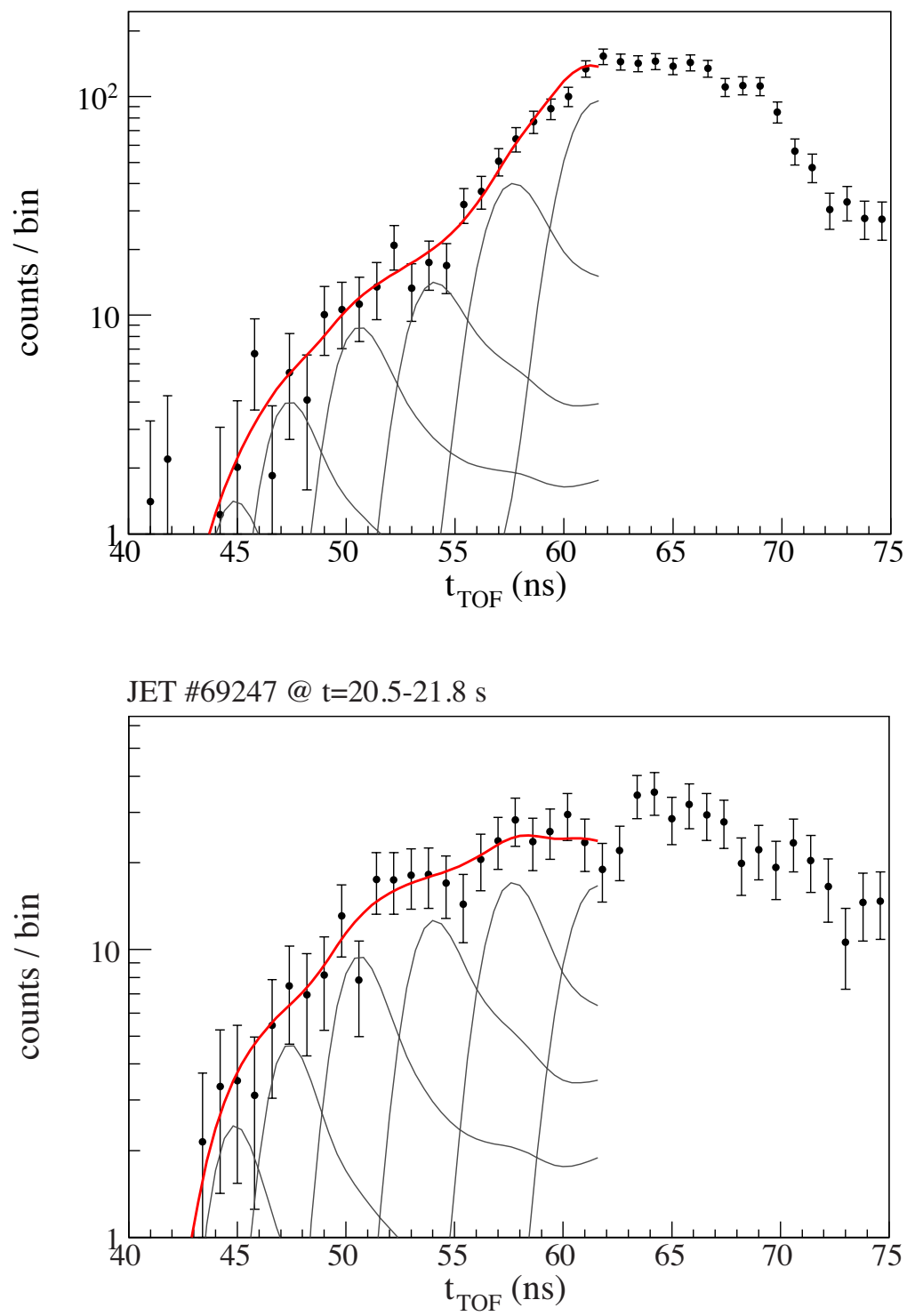

Figure 3. Examples of TOFOR data together with fitted $\delta$-spectra (grey lines). The summed neutron spectrum component is shown in solid red. Time intervals (4) and (5) are shown in (a) and (b), respectively (c.f. figure 2). 

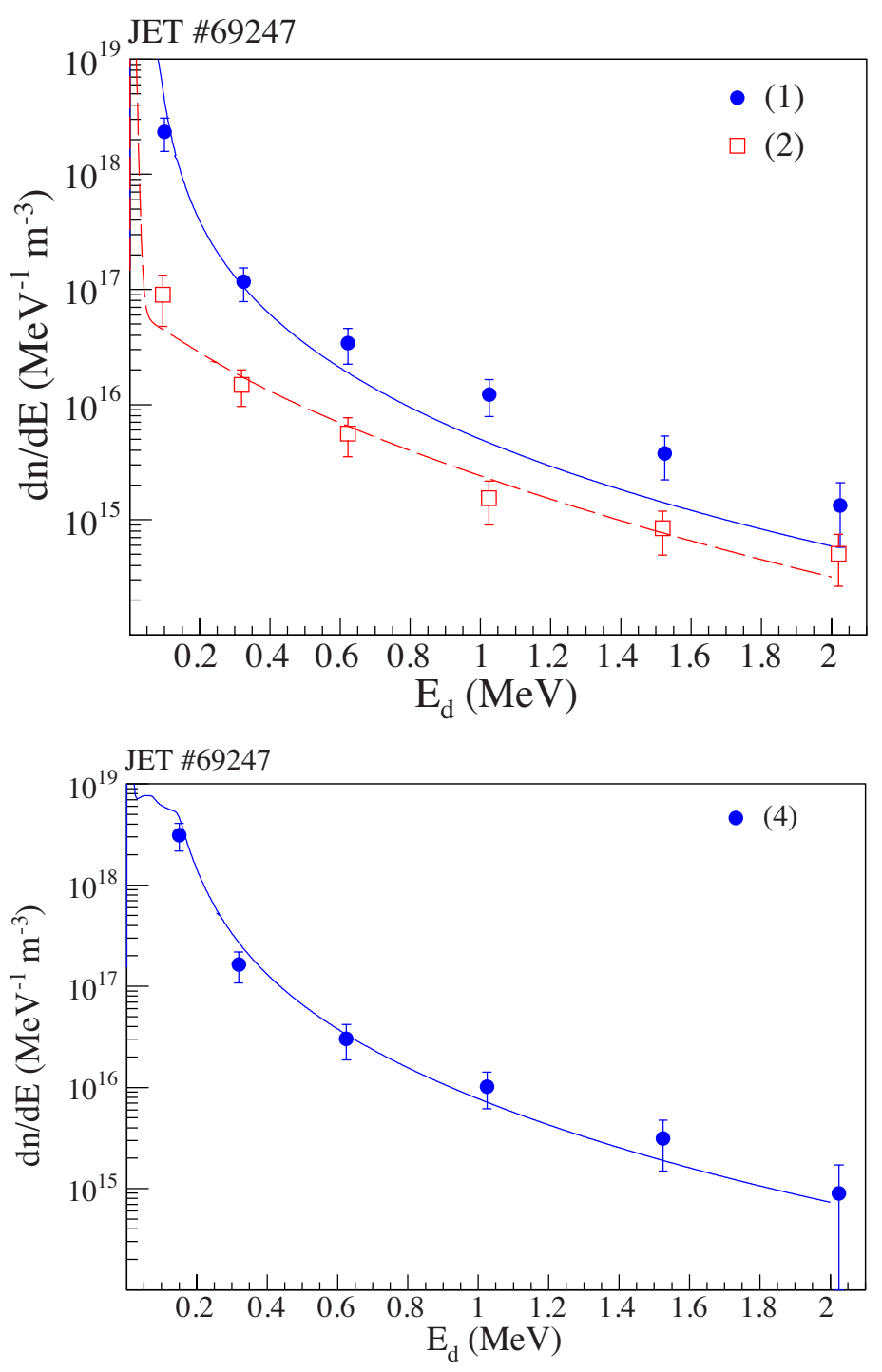

Figure 4. Fast deuterium distributions derived from TOFOR data (points with error-bars) as well as PION simulations for the same time periods (lines). Full drawn lines as well as solid circles represent the phases of the combined NBI+ICRF heating periods (time windows 1 and 4) and the dashed line and hollow squares represent the period with ICRF heating alone (time window 2).

In figures 4, 5 and 6 we show the fast deuterium distributions inferred from the TOFOR data together with the distributions calculated by PION. Note that TOFOR measures a line-integrated neutron flux, and the distributions obtained from the TOFOR data therefore represent the average condition in the core plasma. For this reason we compare the TOFOR results with the PION results averaged in the core plasma, here taken as the innermost $5 \mathrm{~m}^{3}$.

Figure 4 illustrates the conditions during combined NBI+ICRF heating as well as ICRF alone. The neutron rates are here relatively stable during these time steps. In the upper panel the distribution during the first beam blip (time window 1) as well as between the beam blips (time window 2) are shown, and in the lower panel we show the distribution during the second NBI blip (step 4). The deuterium distributions derived from TOFOR data are shown as points with error-bars (solid circles for 
NBI+ICRF and hollow squares for ICRF alone), and the simulations using PION are shown as lines (full drawn for NBI+ICRF and dashed for ICRF alone).

Starting with the combined NBI+ICRF heating, two distinct features of the distribution can be seen. At lower energies, just above the maximum NBI injection energy of about $100 \mathrm{keV}$, the distribution decreases rapidly. Around $0.5 \mathrm{MeV}$, the distribution then gradually flattens out and approaches a Maxwellian shape, which at these energies is a straight line in a logarithmic plot. On the other hand, in the case of ICRF heating alone, the distribution takes on a Maxwellian-like shape already from the first analyzed energy bin (here $100 \mathrm{keV}$ ). The difference between combined NBI+ICRF heating and ICRF heating alone is here seen to be manifested mainly at energies lower than $0.5 \mathrm{MeV}$. At higher energies all distributions take on the same Maxwellian-like shape. And in all cases, the temperature of the Maxwellian is around $350 \mathrm{keV}$.

Comparing the distributions from TOFOR data with the PION simulations we see that the qualitative features of the different heating schemes are well reproduced by the PION simulations. The PION simulation suggests that the power absorbed by deuterium increases three to four-fold, from $2 \%$ of the total power when ICRF heating is used alone to about 6-8 \% during the periods of combined heating. Throughout the discharge, hydrogen minority heating remains the main absorption mechanism, absorbing about $88-94 \%$ of the total ICRF power, while direct electron damping is weaker and absorbs about $4 \%$ of the total ICRH power. Given the good agreement between the distributions from TOFOR and PION when comparing ICRF only with $\mathrm{NBI}+$ ICRF heating, we conclude that the variation in power partition to deuterium is well modeled by PION.

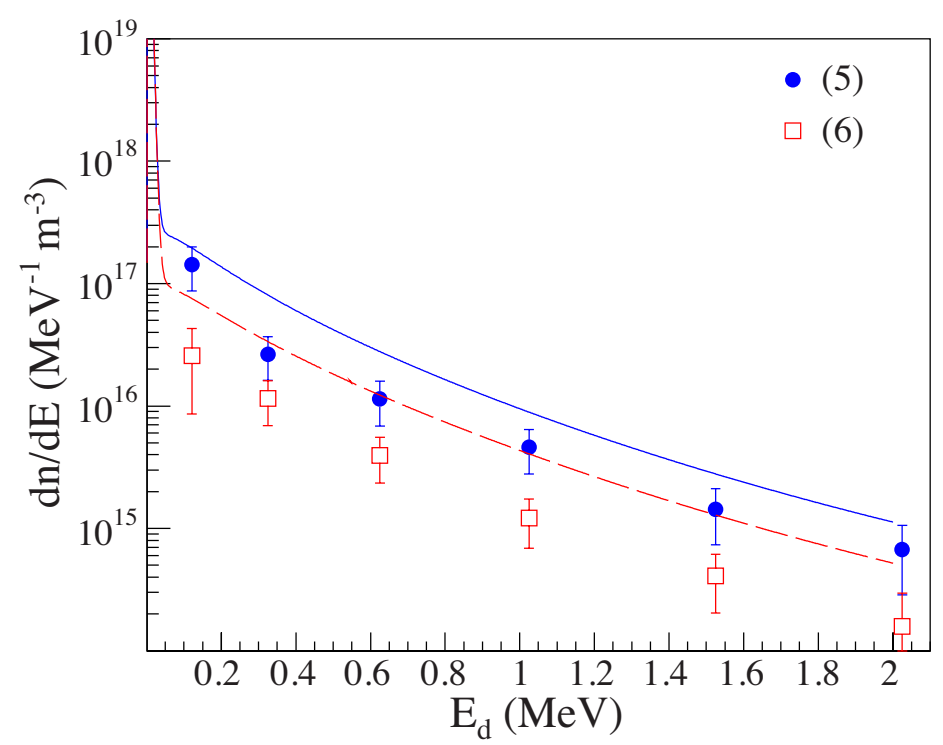

Figure 5. Fast deuterium distributions derived from TOFOR data (points with error-bars) as well as PION simulations for the same time periods (lines) for time steps (5) and (6), c.f. figure 2.

Continuing with figure 5, the distributions for time windows 5 and 6 are compared. These represent a phase where the NBI heating has been switched off and the fast deuterium distribution is gradually decaying. From both the measured distributions as 
well as from those calculated with PION it is seen that the temperature (slope) stays constant, and it is only the amplitudes of the distributions that are decreasing. Although the slope of the modeled distribution agrees well with the one measured, we see that the amplitude of the measured distribution is systematically lower by a factor of two.

This is most likely due to the two monster sawtooth crashes observed at $\mathrm{t}=20.5$ and $21.8 \mathrm{~s}$. Even if sawteeth activity is not expected to result in any significant redistribution of trapped ICRF heated ions in the $\mathrm{MeV}$ range, it is expected to redistribute the passing ions in the NBI slowing down population [Jaulmes 2014], which here serves as a seed for the ICRF heating. These sawteeth are not a part of the PION model used here, and therefore the modeling does not capture this effect.

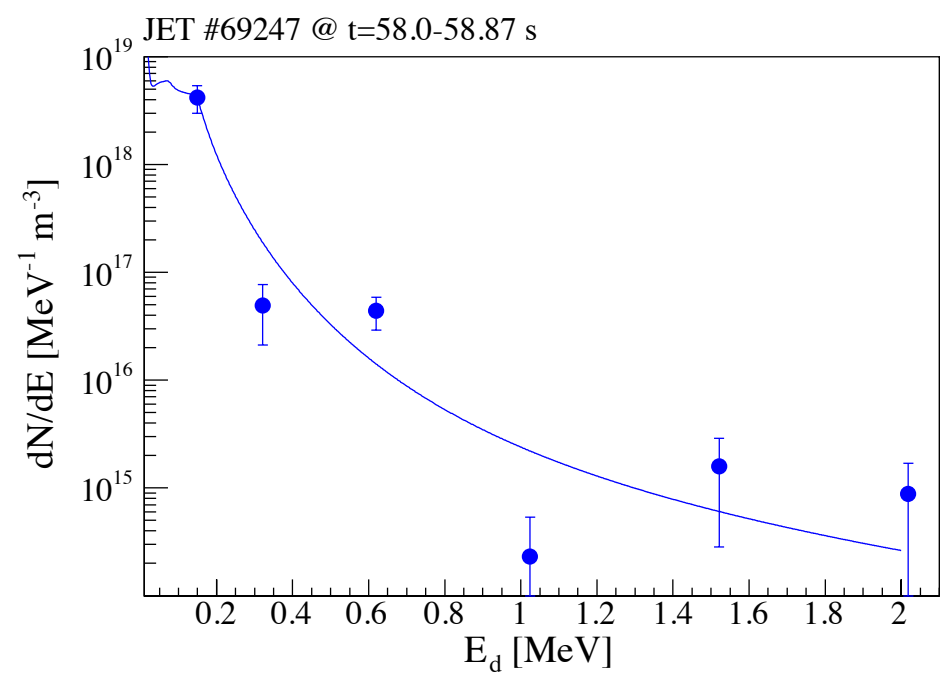

Figure 6. Fast deuterium distribution derived from TOFOR data for step 3 (points with error-bars) as well as PION simulations.

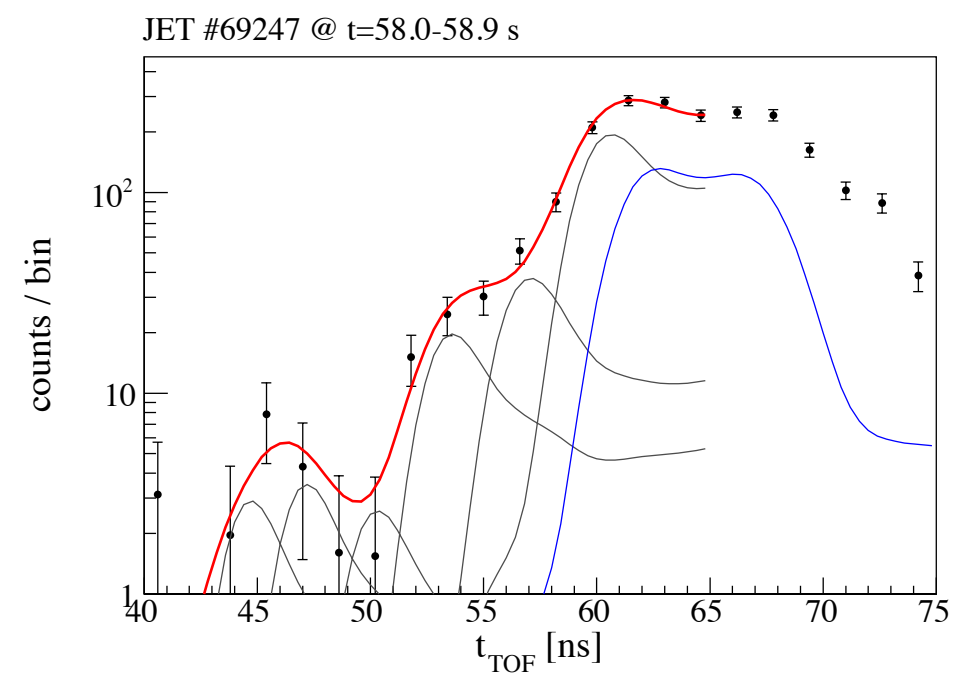

Figure 7. TOFOR data together with fitted $\delta$-spectra (grey lines) for step 3.

Finally, the distribution function during the onset of the second NBI blip (time window 3 ) is shown in figure 6 . In contrast to all other time windows studied above, 
an inverted shape is seen here with a conspicuous dip for deuterons around $1 \mathrm{MeV}$. The agreement with the PION simulation is here very poor. Looking at the raw TOFOR data shown in figure 7, we clearly see the signs of the dip around $t_{\mathrm{TOF}}=50 \mathrm{~ns}$. Compared with the data shown in figure 3 (a) the effect is significant. In fact, when inverting the distribution function from the TOFOR data, the bin at $E_{\mathrm{d}}=1 \mathrm{MeV}$ cannot even be statistically separated from zero.

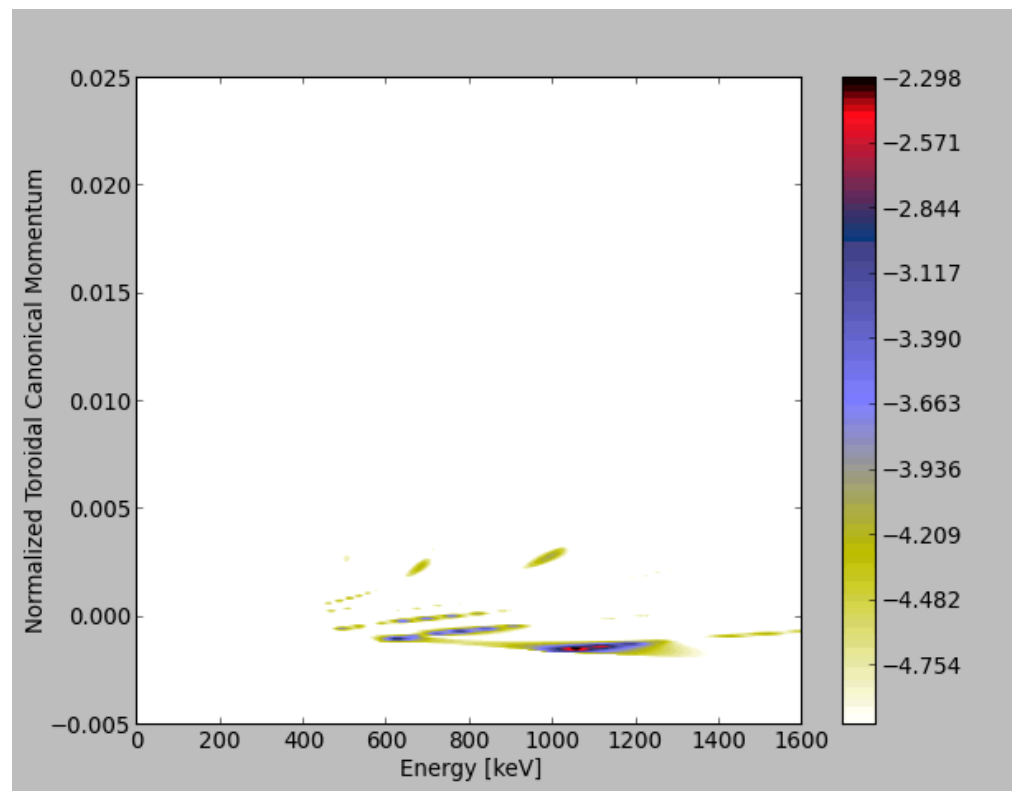

Figure 8. Location and energy of energetic deuterons interacting with the $n=7$ tornado mode observed in time window 3 .

It seems natural to consider the possibility of MHD activity, and Toroidal Alfvén Eigenmodes (TAEs) in particular, to be the cause of the unexpected energy distribution of fast ions measured by TOFOR in time window 3 . It is well known that TAEs may interact with fast ions and they were also observed throughout the entire heating phase of JET discharge \#69247, with toroidal mode numbers $n=3,4$ and 5 . Tornado modes, i.e., TAEs inside the $q=1$ surface, were also observed in two groups around $t=14$ and $t=19 \mathrm{~s}$, with toroidal mode numbers $\mathrm{n}=5,6$ and 7 . The second group of tornado modes around $t=19 \mathrm{~s}$ were excited during the period corresponding to time window 3, which is precisely the time window for which the inverted distribution in energy was measured.

Tornado modes at JET have previously been found to cause a significant redistribution of resonant fast ions, moving them away from the plasma core [Nabais 2010 and 2012]. The radial displacement of fast ions also implies a non-negligible increase in the normalized magnetic moment [Nabais 2014], which pushes the turning points of trapped particles outwards along the major radius. This can be understood from equation (1) and the fact that $\xi=0$ at the turning point. The effect is that the orbits can potentially be displaced completely out of the TOFOR sight line, see figure 2 as well as the discussion in Hellesen 2010b, and as demonstrated in [J. Eriksson 2013] the TOFOR instrument is indeed very sensitive to the fast ion profile along the tokamak major radius. 
In order to evaluate the possibility of tornado modes being the cause of the inverted fast ion distribution observed in time window 3, the CASTOR-K code [Borba 1999 and Nabais 2015] was used to identify the energies and the locations of the resonant ions. CASTOR-K used the distribution function calculated with PION as input, as well as the equilibrium calculated by HELENA [Huysmans 1991] and the eigenfunctions calculated by MISHKA [Mikhailovskii 1997].

Indeed, the modeling results suggest that the observed tornado modes interact with core localized energetic ions through resonances, mainly between 0.6 and $1.2 \mathrm{MeV}$, but with the strongest interactions between 1 and $1.2 \mathrm{MeV}$ (see figure 8 for an example of the $n=7$ mode). This is exactly the same energy region in which the inverted distribution was measured. Attempting to calculate the actual redistribution in detail is out of the scope of this paper, and a more detailed examination of the phenomenon will be the topic of forthcoming work. However, we conclude that the tornado modes are likely to be the cause of the inverted distribution since we have a correlation both in time and energy as well as a mechanism that can transport the ions out of the TOFOR sight line.

Tornado modes, due to their location in the plasma core, may not necessarily lead to strong signals in the Mirnov coils in contrast to global TAEs, which extend to the plasma edge. The centralized locations of the tornado modes also means that their interactions might not result in lost ions, but rather a displacement to confined orbits further out in the plasma. This is also in line with data from lost ion measurements, were no change in the lost ion pattern was observed during time window 3.

\section{Conclusions}

The efficiency of harmonic ICRF heating increases with the temperature of the resonant ion species. This is important for burning plasma operations with ICRF heating since an efficient harmonic ICRF heating of a fuel ion species is much preferred to introducing a minority ion species, which dilutes the plasma and reduces fusion power. In this paper we have studied this increased efficiency in fusion plasmas at the Joint European Torus (JET) in a controlled way by using the slowing down distribution from NBI heating to simulate the effects of a high temperature plasma. The fast deuterium distributions were inferred using data from the neutron time of flight spectrometer TOFOR. We analyzed a plasma that was heated with ICRF at a constant power, but with a modulated NBI heating. This allowed us to dynamically study how the fast ion distribution responds to the NBI heating being switched on and off.

During periods of combined NBI+ICRF heating, the measured distributions between 100 and $500 \mathrm{keV}$ are elevated by up to 10 times compared to periods with ICRF heating alone. This is well reproduced by the PION modeling, showing that the increasing efficiency of harmonic heating ( 3 to 4 times absorbed power) is accurately modeled with the code. From the comparison between modeled and measured fast deuterium distribution functions we can therefore conclude that the modeling of the temperature (energy) dependent power partition for deuterium is correctly modeled in the PION code.

However, during one phase of the discharge, we also observed an inverted distribution function with strong disagreement between the measurement and the 
PION modeling. Resonant interactions with tornado modes, i.e., core localized Toroidal Alfven Eigenmodes have been identified as a likely explanation for the inverted distribution.

\section{Acknowledgements}

This work has been carried out within the framework of the EUROfusion Consortium and has received funding from the Euratom research and training programme 20142018 under grant agreement No 633053. The views and opinions expressed herein do not necessarily reflect those of the European Commission.

\section{References}

Borba 1999, D. Borba and W. Kerner, 1999 J. Comput. Phys. 153101

J. Eriksson 2013, Jacob Eriksson, Carl Hellesen, E Andersson Sundén, Marco Cecconello, Sean Conroy, Göran Ericsson, M Gatu Johnson, SD Pinches, SE Sharapov, Matthias Weiszflog, Plasma Phys. and Contr. Fusion 55 (2013) 015008

L-G. Eriksson 1993, L.-G. Eriksson, T. Hellsten and U. Willén, Nuclear Fusion 33 (1993) 1037

L-G. Eriksson 1998, L.-G. Eriksson, M.J. Mantsinen, F.G. Rimini, F. Nguyen, C. Gormezano, D.F.H. Start and A. Gondhalekar, "ICRF Heating of JET Plasmas with the Third-Harmonic Deuterium Resonance," Nuclear Fusion 38 (1998) 265-278.

L-G. Eriksson 1999, L.-G. Eriksson, M.J. Mantsinen, V.P. Bhatnagar, A. Gondhalekar, C. Gormezano, P.J. Harbour, T. Hellsten, J. Jacquinot, H.J. Jäckel, K. Lawson, C.G. Lowry, E. Righi, G.J. Sadler, B. Schunke, A.C.C. Sips, M.F. Stamp, D.F.H. Start, "Theoretical analysis of ICRF heating in JET DT plasmas," Nuclear Fusion 39 (1999) 337-352.

Gatu 2008, Gatu Johnson M. et al 2008 Nucl. Instrum. Methods A 591417

Hellesen 2010a, Plasma Phys. Control. Fusion 52 (2010)

Hellesen 2010b, Nucl. Fusion 50 (2010) 022001

Hellesen 2013, Nucl. Fusion 53 (2013) 113009

Hellsten 2004, T. Hellsten et al, Nucl. Fusion 44 (2004) 892-908

Huysmans 1991, Huysmans G.T.A., Goedbloed J.P. and Kerner W. 1991 Int. J. Mod. Phys. C 2371

ITER 1999, "ITER Phycics basis, Chapter 6: Plasma auxiliary heating and current drive", Nuclear Fusion, Vol. 39, No. 12

Jaulmes 2014, F. Jaulmes, E. Westerhof and H.J. de Blank, Nucl. Fusion 54 (2014) 104013 (12pp) 
Johnson 2016, T. Johnson, personal communication.

Kiptily 2005, V.G. Kiptily et al. Nucl. Fusion 45 L21

Mantsinen 1999, M.J. Mantsinen, L.-G. Eriksson, V. Bhatnagar, G. Cottrell, A. Gondhalekar, C. Gormezano, R. König, P. Lomas, E. Righi, F. Rimini, G. Sips, D. Start, F.X. Söldner, D. Testa, B. Tubbing and K.-D. Zastrow, "Analysis of Bulk Ion Heating with ICRH in JET High Performance Plasmas," Plasma Physics and Controlled Fusion 41 (1999) 843-865

Mantsinen 2002a, M.J. Mantsinen et al. Physical Review Letters 88 (2002) 105002:1-4

Mantsinen 2002b, M. J. Mantsinen, C. C. Petty, L.-G. Eriksson, T. K. Mau, R. I. Pinsker, and M. Porkolab, "Analysis of combined fast wave current drive and neutral beam injection in the DIII-D tokamak"

Mantsinen 2016, http://ocs.ciemat.es/EPS2016ABS/pdf/P1.035.pdf

Mikhailovskii 1997, Mikhailovskii A.B., Huysmans G.T.A., Kerner W.O.K. and Sharapov S.E. 1997 Plasma Phys. Rep. 23844

Nabais 2010, F. Nabais et al. 2010, Nucl. Fusion, 50: 115006

Nabais 2012, F. Nabais et al. 2012, Nucl. Fusion, 52: 083021

Nabais 2014, F Nabais et al. 2014, Nucl. Fusion, 54: 104011

Nabais 2015, Nabais F., Borba D., Coelho R., Figueiredo A., Ferreira J., Loureiro N. and Rodrigues P. 2015 Plasma Sci. Technol. 1789

Salewski 2017, M. Salewski, M. Nocente, A.S. Jacobsen, F. Binda, C. Cazzaniga, G. Ericsson, J. Eriksson, G. Gorini, C. Hellesen, A. Hjalmarsson, V.G. Kiptily, T. Koskela, S.B. Korsholm, T. Kurki-Suonio, F. Leipold, J. Madsen, D. Moseev, S.K. Nielsen, J. Rasmussen, M. Schneider, S.E. Sharapov, M. Stejner, M. Tardocchi "MeV-range velocity-space tomography from gamma-ray and neutron emission spectrometry at JET”, Nucl. Fusion 57056001

Start 1999, D.F.H. Start, et al., "Bulk ion heating with ICRH in JET DT plasmas," Nuclear Fusion 39 (1999) 321-336. 